\title{
Zustand nach Sectio: Management und bildgebende Diagnostik
}

Janine Hoffmann, Holger Stepan

\section{Übersicht}

Einleitung

275

Sekundäre Sectio-Komplikationen

bei Folgeschwangerschaften

276

Geburtsplanung nach

deutschen Leitlinien

Pränatale Betreuung nach

deutschen Leitlinien

\section{Einleitung}

Aufgrund steigender Sectio-Raten, insbesondere in den Industrieländern, stellen sich heute immer mehr Frauen mit wenigstens einer Sectio in der Anamnese zur Geburtsplanung vor. Waren es laut Statistischem Bundesamt im Jahr 1993 in Deutschland lediglich 16,9\%, so entbanden im Jahr 2003 bereits 25,5\% und im Jahr 2013 bereits 31,8\% aller Frauen mittels einer Sectio. Ein Grund für die ansteigenden Sectio-Raten ist der Wunsch nach Sicherheit für Mutter und Kind und nicht zuletzt die gute Planbarkeit der Methode. Da primäre Komplikationen nur selten auftreten, genießt die operative Entbindung durch eine Sectio Vertrauen bei Ärzten und Patientinnen. Transfusionsbedürftige Blutungen (1,3\%) und Wundinfektionen mit Wundheilungsstörungen (6\%) zählen dabei zu den häufigsten perioperativen Komplikationen [1].

Neben den meist gut beherrschbaren primären Komplikationen erfordern sekundäre Komplikationen in Folgeschwangerschaften immer häufiger eine entsprechende geburtsmedizinische Diagnostik und Therapie. $\mathrm{Zu}$ den wesentlichen Sekundärkomplikationen zählen:

- Implantations- und Nidationsstörungen

- Plazentationsstörungen

- Defekte der Uterotomienarbe
Die hiermit verbundenen, nicht selten lebensbedrohlichen Risiken für Mutter und Kind erfordern ein adäquates geburtsmedizinisches Management. Dieses umfasst die pränatale Diagnostik, eine intensive Geburtsplanung und eine angemessene Geburtsleitung. Hierbei ist u. a. zu berücksichtigen, dass die Risiken für Sekundärkomplikationen mit jedem erneuten Kaiserschnitt zunehmen.

Die Indikation zur Re-Sectio sollte insbesondere bei weiter bestehendem Kinderwunsch streng gestellt werden.

Der hohe Stellenwert der Schwangerenbetreuung bei Z.n. Sectio macht die Auseinandersetzung mit diesem wichtigen geburtsmedizinischen Thema dringend notwendig. Dieser Artikel soll dem Leser die wichtigsten Komplikationen bei Z.n. Sectio und den optimalen Ablauf der Schwangerenbetreuung und Geburtsleitung bei Z.n. Sectio vermitteln. 


\section{Sekundäre Sectio-Komplikatio- nen bei Folgeschwangerschaften}

\section{Narbenschwangerschaften}

\section{Definition und Inzidenz}

Eine besondere Form der ektopen Schwangerschaften ist die Narbenschwangerschaft. Die Implantation der Blastozyste findet hierbei direkt im Narbenbereich statt. Mit zunehmendem Schwangerschaftsalter steigt das Risiko für eine Uterusruptur und damit auch das Risiko für eine lebensbedrohliche maternale Blutung. Neben anderen transmuralen Uterusoperationen ist der Z.n. Sectio der wichtigste Risikofaktor für das Auftreten von Narbenschwangerschaften [2]. Eine Narbenschwangerschaft betrifft 0,15\% der Frauen mit Z.n. Sectio und macht 6,1\% aller ektopen Schwangerschaften bei Patientinnen mit wenigstens einer Sectio in der Anamnese aus. Mit einer Inzidenz von 1:2226-1:1800 Schwangerschaften im 1. Trimenon sind Narbenschwangerschaften zwar selten, doch ist bei zunehmender Sectio-Rate von einer steigenden Inzidenz auszugehen [3,4]. Ob das Auftreten von Narbenschwangerschaften mit der Anzahl der Kaiserschnitte in der Vergangenheit korreliert oder nicht, ist noch nicht eindeutig belegt.

\section{Diagnostik}

Kennzeichnend ist die Einnistung der Schwangerschaft direkt im Narbenbereich. Für die primäre Diagnostik ist die Sonografie die Methode der Wahl. Insbesondere bei unklaren Befunden oder auch zur OP-Planung sollte die Diagnostik mit einer MRT vervollständigt werden.
Therapiealternativen bei
Narbenschwangerschaft

Neben den bisherigen Behandlungsmöglichkeiten bei Narbenschwangerschaft (Exzision, Hysterektomie, Methotrexat-Gabe), werden neuere, alternative Behandlungsalternativen, z. B. auf Embolisation der A. uterina basierende Strategien [6] oder die Applikation von „High Intensity focused Ultrasound“ (HIFU) [7] diskutiert, sie haben sich in der Praxis bisher aber noch nicht durchgesetzt.

Je früher eine Narbenschwangerschaft diagnostiziert wird, desto besser ist das Outcome für die Frau. Deshalb sollte die Implantationsstelle des Embryos bei Patientinnen mit Z.n. Sectio so früh wie möglich in der Frühschwangerschaft identifiziert werden.

Abbildung 1 zeigt einen typischen Befund einer Narbenschwangerschaft im Ultraschall und MRT.

\section{Therapie}

Bei Vorliegen einer Narbenschwangerschaft ist ein Schwangerschaftsabbruch indiziert. Aufgrund der geringen Inzidenz sind einheitliche Behandlungsstandards bis heute nicht verfügbar. Prinzipiell stehen operative Maßnahmen zur Exzision der Schwangerschaft oder die Hysterektomie sowie medikamentöse Behandlungsstrategien mit Methotrexat lokal, systemisch oder kombiniert zur Verfügung $[4,5]$. Wegen der guten Chancen für einen Fertilitätserhalt und der geringeren Invasivität wird die medikamentöse Therapie in vielen Zentren bevorzugt.
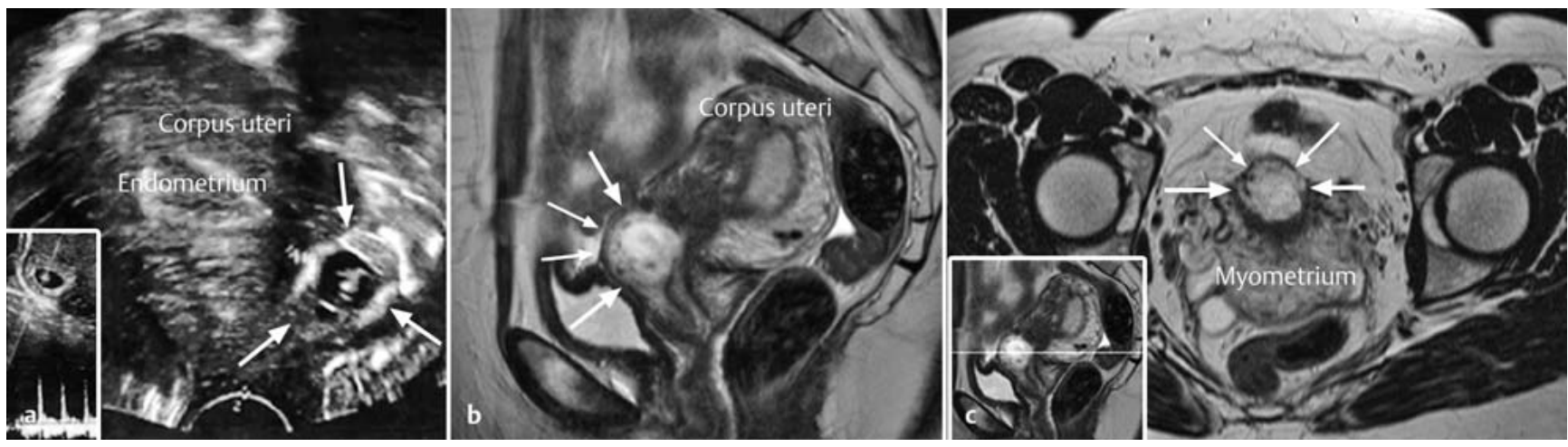

Abb. 1 Narbenschwangerschaft: Sonografie (a) und MRT (b, c) einer intakten (Box 1 a, Dopplersonografie) Narbenschwangerschaft in der rechnerisch 11. SSW. Im Bereich des unteren Uterinsegments ist ein Fruchtsack mit einem Embryo (dicke Pfeile) erkennbar. Sowohl sonografisch als auch in der MRT zeigt sich die Serosa intakt (dünne Pfeile), kein Hinweis für eine Ruptur. Das Myometrium des unteren Uterinsegments ist aber nahezu vollständig aufgebraucht. Bei dieser Patientin wurde aufgrund der fortgeschrittenen Schwangerschaft und der hiermit verbundenen Rupturgefahr eine Hysterektomie durchgeführt. 


\section{Plazentationsstörungen}

\section{Definition und Inzidenz}

Plazentationsstörungen sind ebenfalls selten und manifestieren sich als

- Lageanomalien (tiefreichende Plazenta, Placenta praevia) oder

- Plazentainvasionsstörungen (Placenta accreta, Placenta increta oder Placenta percreta).

Plazenta-Invasionsstörungen entstehen durch das im Narbenbereich fehlende Stoppsignal durch die Decidua basalis.

Die Einteilung der verschiedenen Schweregrade erfolgt anhand der Invasionstiefe.

Während bei der Placenta accreta nur die Decidua basalis infiltriert wird, beschreibt die Placenta increta eine Invasionstiefe ins Myometrium bis an die Serosa heran. Bei der seltensten, aber gleichsam schwersten und gefährlichsten Form der Plazentationsstörung, der Placenta percreta, durchwächst die Plazenta die dünne Serosa. Nicht selten sind die eng benachbarten Strukturen wie Harnblase, Nerven und Gefäße einbezogen.

\section{Tipp für die Praxis}

Häufig sind Lageanomalien der Plazenta mit Invasionsstörungen assoziiert. Bereits ein Kaiserschnitt in der Anamnese erhöht das Risiko für Plazentationsstörungen in der Folgeschwangerschaft. Jeder weitere Kaiserschnitt erhöht das Risiko für Plazentationsstörungen in den Folgeschwangerschaften weiter.

Die Inzidenz einer Placenta accreta/increta/percreta steht im engen Zusammenhang mit dem gleichzeitigen Vorliegen einer Placenta praevia (s.Tab. 1).

\section{Diagnostik}

Alle Plazentationsstörungen sind mit einem hohen Risiko für transfusionsbedürftige Blutungen und Organverletzungen sowie der Notwendigkeit einer notfallmäßigen Hysterektomie verbunden. Nach deutschen Leitlinien sollte die sorgfältige Beschreibung der Plazentalokalisation mittels der Sonografie erfolgen [10]. Liegt die Plazenta im Narbenbereich, wurde ein tiefer Plazentasitz oder eine Placenta praevia beschrieben, so ist auch eine sonografische Diagnostik der Invasionstiefe unter Zuhilfenahme des Farbdopplers erforderlich.

\section{Tabelle 1}

Inzidenzen für das Risiko zur Entwicklung einer Placenta praevia sowie einer Placenta accreta/increta/percreta in Abhängigkeit vom Vorliegen einer Placenta praevia und der Anzahl der vorangegangenen Sectiones (nach $[8,9]$ ).

\begin{tabular}{llll}
$\begin{array}{l}\text { stattgehabte } \\
\text { Sectiones }\end{array}$ & $\begin{array}{l}\text { Inzidenz für Placenta } \\
\text { praevia in der } \\
\text { Folgeschwangerschaft }\end{array}$ & $\begin{array}{l}\text { Risiko für Placenta accreta/ } \\
\text { increta/percreta }\end{array}$ \\
\hline 1 & $0,8 \%$ & $\begin{array}{l}\text { ohne Placenta } \\
\text { praevia }\end{array}$ & $\begin{array}{l}\text { mit Placenta } \\
\text { praevia }\end{array}$ \\
\hline 2 & $0,03 \%$ & $3,3 \%$ \\
\hline 3 & $2 \%$ & $0,2 \%$ & $11 \%$ \\
\hline
\end{tabular}

Insbesondere bei zweifelhaften Befunden sollte zusätzlich zur Sonografie eine MRT erfolgen $[11,12]$.

Abbildung 2 zeigt typische Befunde einer Placenta increta und Abbildung 3 typische Befunde einer Placenta percreta.

Sonografie. Bei der Diagnostik der Placenta accreta/increta/percreta steht die 2-D-/3-D- und farbkodierte Dopplersonografie im Vordergrund. Aufgrund ihrer einfachen Verfügbarkeit und ihrer hohen diagnostischen Wertigkeit ist sie die Screeningmethode der Wahl.

MRT. Auch die MRT hat als komplementäres Untersuchungsverfahren einen bedeutenden festen Stellenwert in der pränatalen Diagnostik von Plazentationsstörungen. Sie ist hauptsächlich erforderlich, wenn die Diagnose im Ultraschall nicht eindeutig möglich ist.

Die Diagnosekriterien der MRT entsprechen weitestgehend denen der Sonografie. Neben der Ausdünnung des Myometriums und der Unterbrechung der Grenz-

\section{Sonografische Diagnosekriterien}

- Ausdünnung des Myometriums bei fehlender Abgrenzung zwischen Plazenta und Myometrium

- intraplazentare Lakunenbildung

- Hypervaskularisation des subplazentaren Raumes

- eine senkrecht zum Myometrium verlaufende Vaskularisation

Nicht selten ist eine Vorwölbung der uteroplazentaren Fläche in die Harnblase (Bulging) erkennbar (s. Abb. 2 und 3) [13]. 


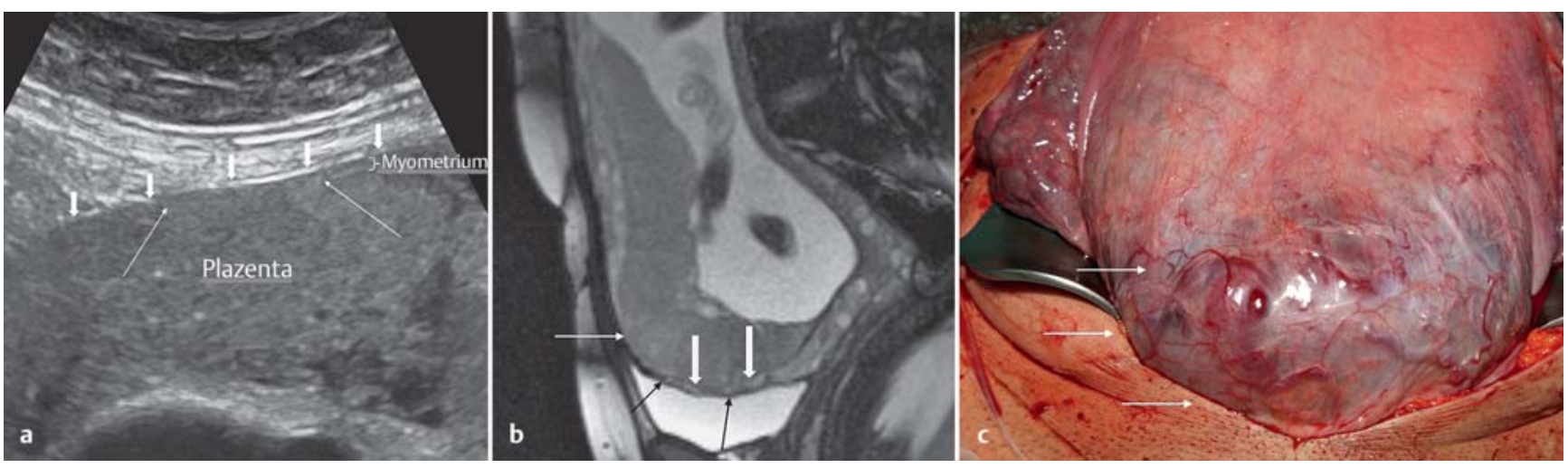

Abb. 2 Placenta increta: Sowohl sonografisch (a) als auch in der MRT (b) können Plazenta und Myometrium im inkreten Bereich (dünne, weiße Pfeile) nicht mehr abgegrenzt werden. Die Plazenta infiltriert das Myometrium tief. Die sehr zarte Serosa (dicke, weiße Pfeile) erscheint MRT- und sonomorphologisch intakt. Die Harnblasenwand erscheint in der MRT glatt abgrenzbar, über die gesamte Breite erhalten und somit nicht infiltriert (b, dünne, schwarze Pfeile). Intraoperativ bestätigte sich der bildgebende Verdacht einer Placenta increta (c, dünne, weiße Pfeile) (Quelle der MRT-Bilder: Klinik und Poliklinik für Diagnostische und Interventionelle Radiologie der Universität Leipzig).
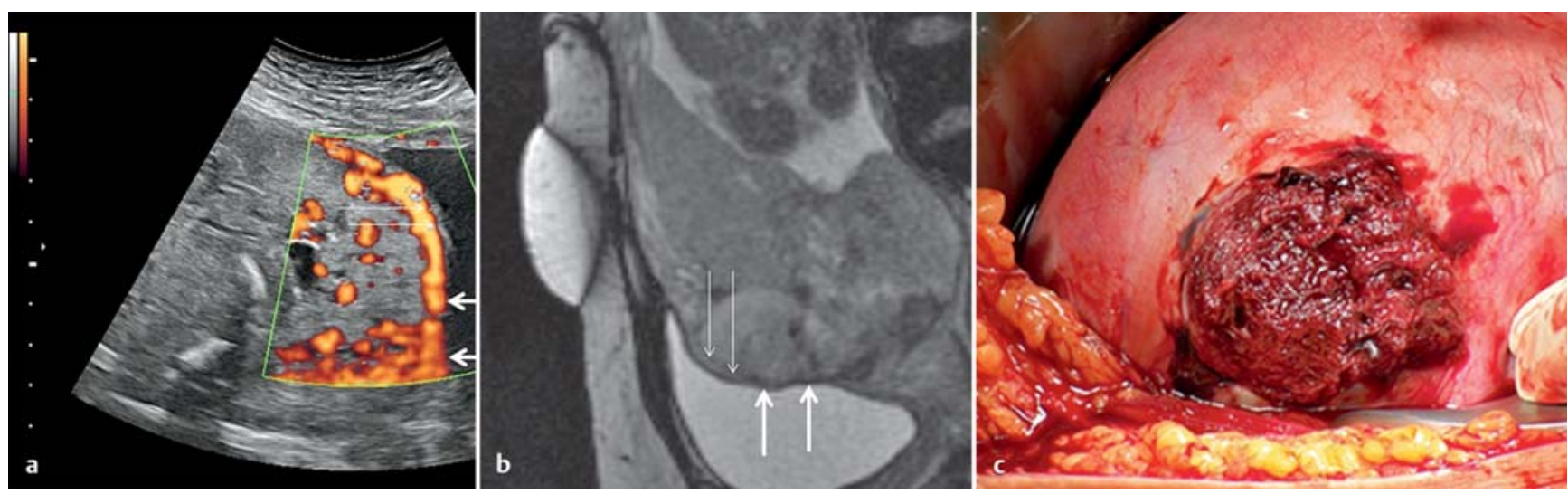

Abb. 3 Placenta percreta: Sowohl sonografisch (a) als auch in der MRT (b) erscheint die Plazenta über die Serosa hinaus bis an die Harnblase heran (dünne Pfeile) und zum Teil auch in die Muscularis der Harnblase hinein zu wachsen (dicke Pfeile). Intraoperativ bestätigte sich der bildgebende Verdacht einer Placenta percreta (c) (Quelle der MRT-Bilder: Klinik und Poliklinik für Diagnostische und Interventionelle Radiologie der Universität Leipzig).

fläche zwischen Plazenta und Myometrium ist die Darstellung dunkler, intraplazentar verlaufender Linien in der T2-Wichtung typisch. Auch in der MRT sind Hypervaskularisation und Lakunenbildung sowie ein Bulging hinweisend auf das Vorliegen von Plazenta-Invasionsstörungen [13].

Sensitivität und Spezifität. Neuere Metaanalysen zur Genauigkeit der Sonografie und MRT zeigen eine hohe Sensitivität und Spezifität für beide Methoden. Für die Sonografie sind Sensitivitäten von $83-90,7 \%$ beschrieben, für die MRT $82-94,4 \%$. Die Spezifitäten sind für die Sonografie mit 95-96,9\% und mit 84-88\% für die MRT angegeben [14-16].

\section{Therapie}

Betroffene Patientinnen müssen ausführlich über das erhöhte Blutungsrisiko und die Notwendigkeit einer erneuten Sectio aufgeklärt werden. Zudem muss mit der Patientin besprochen werden, dass die Wahrscheinlichkeit für die Notwendigkeit einer Hysterektomie oder weiterer medikamentöser (Methotrexat), chirurgischer (z. B. bei Verletzung umliegender Strukturen) oder radiologischer (Embolisation der Aa. uterinae) Behandlungsmaßnahmen hoch ist. Die Erstvorstellung in der Entbindungsklinik sollte bis zur 30. SSW erfolgen. Wegen des hohen Blutungsrisikos sollte die Patientin präpartal hospitalisiert und die primäre ReSectio nach der vollendeten 34. SSW geplant werden [12]. 


\section{Tipp für die Praxis}

Die Betreuung von Patientinnen mit Plazentationsstörungen sollte ausschließlich an einem geeigneten Perinatalzentrum mit einer Blutbank erfolgen [12].

\section{Narbendehiszenzen}

\section{Definition und Inzidenz}

Narbendehiszenzen müssen klar von Uterusrupturen unterschieden werden. Eine Narbendehiszenz beschreibt lediglich ein Auseinanderweichen des Myometriums im uterinen Nahtbereich.

Bei einer Narbendehiszenz kommt es nicht zu einer Verbindung zwischen Uterushöhle und Bauchhöhle.

Da die Symptome meist gering ausgeprägt sind oder vollständig fehlen, ist die Inzidenz nicht zuverlässig zu ermitteln. Eine Narbendehiszenz wird nicht selten zufällig bei einer Re-Sectio diagnostiziert, welche aus anderen Gründen indiziert wurde. Für Mutter und Kind ist sie vor allem deshalb bedeutsam, weil sie das Risiko für eine lebensgefährliche Uterusruptur erhöht [1719].

\section{Diagnostik}

Sonografie des unteren Uterinsegments. Die Sonografie des unteren Uterinsegments wird in den letzten Jahren zunehmend für die Risikoselektion bei Z.n. Sectio eingesetzt. Wenn auch kontrovers diskutiert, gibt es Studienergebnisse, die darauf hinweisen, dass das Risiko für eine Uterusruptur mit abnehmender Uteruswanddicke im Narbenbereich zunimmt $[17,18]$. Die Messung kann transabdominal oder transvaginal erfolgen. Es kann entweder die Dicke des Myometriums oder die Dicke des gesamten unteren Uterinsegments bestimmt werden (Abb. 4).

Aufgrund der ungenügenden Datenlage gibt es bis heute keine allgemeingültigen Grenzwerte. In einer Metaanalyse aus dem Jahr 2013 wurden die Ergebnisse von 21 Studien ausgewertet. Ein signifikant geringeres Uterusrupturrisiko wurde für die Dicke des Myometriums von 2,1-4,0 mm sowie für die Dicke des gesamten unteren Uterinsegments von 3,1-5,1 mm ermittelt [19]. Wegen der Heterogenität der meist nicht randomisierten Studien und der geringen Fallzahlen können diese Ergebnisse nur als grob orientierend gelten.

Die bedeutenden Limitationen der sonografischen Messung des unteren Uterinsegments sind die Abhängigkeit von Untersucher und Untersuchungsbedingungen. Die vaginale Messung scheint dabei die genauere Messmethode zu sein [20]. Trotz dieser Einschränkungen bietet die Sonografie eine Möglichkeit, die Uteruswand zu beurteilen. Bei kritischer Bewertung und im klinischen Kontext gesehen, kann sie als bildgebende Methode der ersten Wahl bei der Geburtsplanung hilfreich sein.

MRT. Die MRT hat bei der Beurteilung der Sectio-Narbe bisher keine Bedeutung im klinischen Alltag. Sie ist der Sonografie in der räumlichen und zeitlichen Auflösung unterlegen, bietet aber die Möglichkeit, den gesamten Uterus unabhängig von der Erfahrung des Untersuchers und der Patientenkonstitution abzubilden. Ein möglicher zusätzlicher diagnostischer Nutzen der MRT
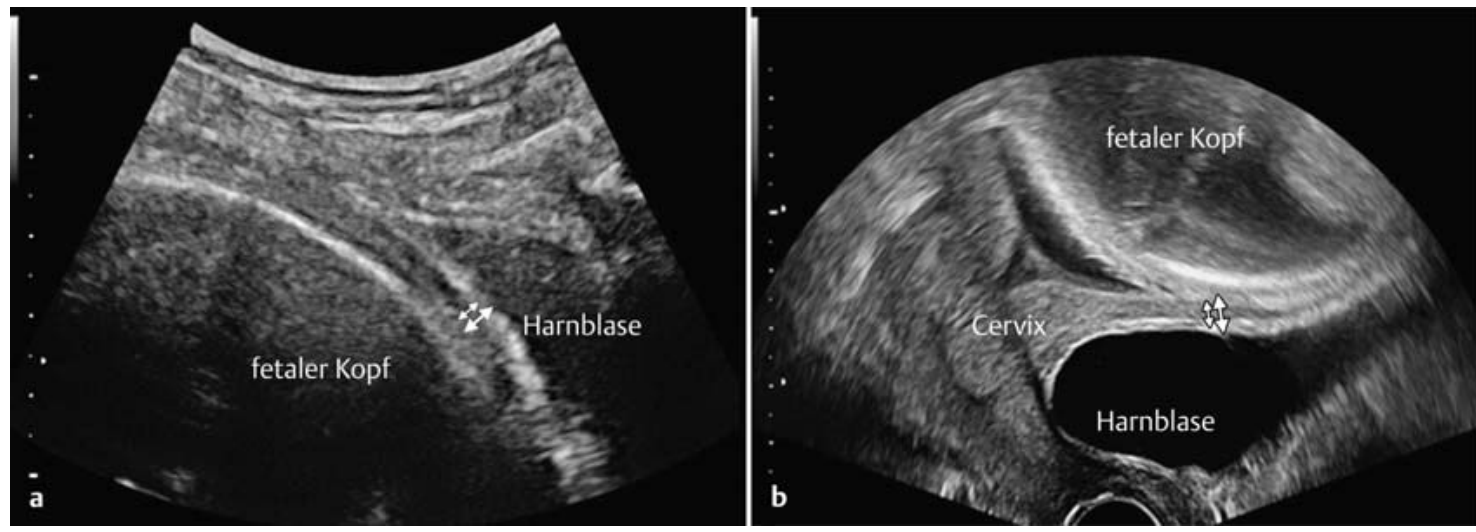

Abb. 4 Sonografie des unteren Uterinsegments: Anhand eines sagittalen Schnittes durch das untere Uterinsegment kann die Dicke der Myometriumschicht (kleiner Doppelpfeil) und die Dicke des gesamten unteren Uterinsegments (großer Doppelpfeil) bestimmt werden [21]. Die Untersuchung ist transabdominal (a) und transvaginal (b) möglich. 
bei der Diagnostik der Wandintegrität muss in Studien noch untersucht werden.

\section{Tipp für die Praxis}

Nach den aktuell gültigen Leitlinien sind zusätzliche bildgebende Verfahren zur Beurteilung der SectioNarbe für die Schwangerenbetreuung und die Geburtsplanung nicht gefordert [10].

\section{Uterusrupturen}

\section{Definition und Inzidenz}

Von einer Uterusruptur spricht man, wenn es zum Auseinanderreißen des gesamten unteren Uterinsegments und dadurch zu einer Verbindung zwischen Uterushöhle und Bauchhöhle kommt. Da es bei einer Uterusruptur zu einem akuten starken Blutverlust kommt, sind Mutter und Kind sofort lebensbedrohlich gefährdet. Das Risiko für eine Uterusruptur ist durch das Vorhandensein von Narbendehiszenzen zwar erhöht, kann aber unter geburtsrelevanten Wehen auch bei intakter Uterusnarbe auftreten. Die Evidenz ist hier jedoch gering.

Uterusrupturen sind selten. In der Literatur variieren die Inzidenzen, liegen aber bei spontanem Geburtsbeginn in allen Veröffentlichungen unter $1 \%[22,23]$.

Zwei oder mehr Sectiones erhöhen das Risiko für Uterusrupturen nicht signifikant [24].

Bei Z.n. Sectio ereignet sich eine Uterusruptur meist im Narbenbereich des unteren Uterinsegments als Querruptur. Je nach Art der vorangegangenen Sectio können aber auch längs verlaufende Rupturen auftreten.

Die Rupturgefahr nimmt bei Überdehnung infolge eines unüberwindlichen Geburtshindernisses, z. B. bei Lageanomalien, fetomaternalem Missverhältnis oder

\section{Risiken bei einer Uterusruptur}

\section{Akute Gefahren}

Tod oder Schädigung von Mutter und/oder Kind infolge Anämie/Schock durch

- Rissblutungen in die freie Bauchhöhle

- Einrisse oder Abrisse der uterinen Gefäße bei seitlichem Einriss des Uterus

\section{Späte Gefahren}

Tod oder Schädigung der Mutter durch Infektionen:

- Peritonitis/Sepsis bestimmten fetalen Anomalien (z.B. Hydrozephalus, Tumore) zu.

Die erhöhte perinatale Morbidität und Mortalität sowohl des Feten als auch der Mutter erfordern eine sorgfältige Geburtsplanung [10,22]. Eine ausführliche Anamnese sowie die Einschätzung der fetomaternalen Proportionen durch klinische und bildgebende Untersuchungen sind hierbei ein zentraler Bestandteil. Eine Uterusruptur birgt nicht nur akute, sondern auch späte Gefahren.

\section{Diagnostik}

Narbenrupturen können mit und ohne vorangehende alarmierende Signale auftreten. Da Warnsignale bei Z.n. Sectio häufig fehlen, wird eine Ruptur nicht selten unvorhersehbar zu einem geburtsrelevanten Problem.

Die Uterusruptur wird anhand der Klinik (Symptome, CTG-Veränderungen) diagnostiziert!

Der Sonderfall einer stillen Ruptur tritt vorrangig bei Narbenrupturen auf. Die Ruptur wird erst spät, manchmal erst nach der Geburt, bemerkt, da es nur selten zu CTG-Veränderungen kommt und Symptome häufig fehlen oder nur sehr gering sind. Die Gefahren einer stillen Ruptur betreffen hauptsächlich die Mutter, da Blutungen und eine Anämie häufig erst spät diagnostiziert und behandelt werden.

\section{Warnsignale}

- Zunahme der Wehentätigkeit bis zum Wehensturm bzw. Dauerkontraktionen

- Druckempfindlichkeit und Spannung des Bauches zwischen Nabel und Symphyse

- Erkennbarwerden und Hochsteigen eines Retraktionsringes, der Bandl'schen Furche (entspricht der oberen Grenze des unteren Uterinsegments in Nabelhöhe oder darüber)

- subjektiv stärkster „Zerreiß-Schmerz“

- Unruhe, Blässe, Erhöhung der Pulsfrequenz, (Todes-) Angst

- Muttermund, wenn noch erhalten, infolge der Einklemmung ödematös

- meist federnd dem Becken aufgepresster Kopf/ große Kopfgeschwulst (Zeichen des Missverhältnisses/Austreibungshindernis) 


\section{Symptome einer Uterusruptur}

- schlagartiges Sistieren der Wehen

- Rupturschmerz (Frau bemerkt, dass etwas in ihr zerrissen ist)

- Schock, Anämie (durch schwere innere Blutungen)

- vaginale Blutung

- der vorher aufgepresste Teil des Kindes ist freier oder ganz frei beweglich

- CTG-Veränderungen

Therapie

\section{Tipp für die Praxis}

Bei einer Uterusruptur oder bei Verdacht auf eine Uterusruptur ist immer die sofortige Beendigung der Geburt, meist durch eine Notfall-Sectio indiziert!

- sofortige i.v. Tokolyse (Fenoterol)

- Schockprophylaxe/Behandlung Schockprophylaxe und Behandlung der Blutung bzw. Atonie

- sofortige Indikation zur Laparotomie - unabhängig von der Situation des Kindes, ob lebendig/tot, intrauterin/geboren/in der freien Bauchhöhle

- Übernähen des Defektes; wenn nicht ausreichend oder bei technischen Schwierigkeiten (veränderte Anatomie, starke Blutungen, Hämatome), kann eine Notfallhysterektomie notwendig sein (in ca. 35\% der Fälle)

\section{Pränatale Betreuung nach deutschen Leitlinien}

Schwangere mit Z.n. Sectio müssen aufgrund der o.g. Risiken engmaschig geburtsmedizinisch betreut werden. Dazu gehören eine adäquate pränatale Diagnostik und Therapie sowie die Festlegung des Geburtsmodus an einem entsprechenden Perinatalzentrum.

Die Betreuung der Risikoschwangerschaft sollte an einem Perinatalzentrum erfolgen.

Narbenschwangerschaften sollten möglichst früh, vor der 12. SSW entdeckt und behandelt werden. Die Lokalisation der Plazenta und gegebenenfalls die Invasionstiefe einer Vorderwandplazenta im Narbenbereich müssen beschrieben werden. Bei Vorliegen von Plazentationsstörungen sind die präpartale Hospitalisation der Patientin zu diskutieren und die primäre Re-Sectio ab der vollendeten 34. SSW zu planen.

Narbendehiszenzen können im gesamten Schwangerschaftsverlauf und auch während der Geburt auftreten. Die bildgebende Diagnostik des Narbenbereiches ist nach Mutterschaftsrichtlinien zwar nicht vorgeschrieben, jedoch in der geburtsmedizinischen Praxis immer weiter verbreitet [10].

\section{Geburtsplanung nach deutschen Leitlinien}

Für die Wahl des Geburtsmodus ist eine gesonderte Planung durch die Geburtsklinik erforderlich. Beim Fehlen von Kontraindikationen besteht die Möglichkeit, eine vaginale Entbindung oder eine primäre ReSectio mit der Patientin zu planen, wobei die Wahl des angestrebten Geburtsmodus bei der Schwangeren liegt. Eine ausführliche Aufklärung über alle Vor- und Nachteile, Risiken und Chancen der jeweiligen Geburtsmodi ist obligater Bestandteil der Geburtsplanung.

Durch eine Sectio in der Vergangenheit wird die Schwangerschaft zur Risikoschwangerschaft und die Geburt zu einer Risikogeburt.

\section{Vaginale Entbindung}

Eine Sectio in der Anamnese ist keine absolute Kontraindikation für eine vaginale Entbindung. Die werdende Mutter muss jedoch rechtzeitig, möglichst in der 35. -36. SSW über die Möglichkeiten eines vaginalen Entbindungsversuches sowie über die mit der Uterusnarbe verbundenen Risiken aufgeklärt und beraten werden. Ein ausführliches, wertungsfreies Gespräch mit der Mutter ist erforderlich. Dieses sollte die Einschätzung der individuellen Risiken und Erfolgsaussichten für eine vaginale Entbindung einbeziehen. Ein OP-Bericht muss angefordert werden.

\section{Selektionskriterien}

Bei der Patientinnenselektion für den vaginalen Entbindungsversuch sind die in Tabelle $\mathbf{2}$ aufgeführten Kriterien zu berücksichtigen. 


\section{Tabelle 2}

Die 3 Säulen der Risikoselektion von Patientinnen mit Z.n. Sectio [10].

$\begin{array}{lll}\text { geburtshilfliche Anamnese } & \text { klinische Untersuchung } & \text { Sonografie } \\ \text { - Verlauf früherer Geburten } & \text { - Leopold'sche Handgriffe } & \text { - Kindslage } \\ \text { - Anzahl früherer Sectiones } & \text { - fetomaternale Proportion } & \text { - Kindsgewicht } \\ \text { - Ursachen früherer Sectiones } & \text { - Beckenanomalien } & \text { - Plazentalokalisation } \\ \text { - Schnittführung früherer Sectiones } & \text { - Adipositas } & \text { - Plazentainsertionstiefe } \\ \text { - andere gynäkologische Operationen } & & \end{array}$

\section{Erfolgschancen für eine vaginale Geburt}

Bei entsprechender Selektion sind die Erfolgschancen für eine vaginale Entbindung hoch. Je nach Patientinnenkollektiv sind in der Literatur Erfolgschancen von 50-90\% (Mittel 73\%) nach 1 Sectio und 45-90\% (Mittel $68 \%$ ) nach 2 oder mehr Sectiones in der Vergangenheit beschrieben [10].

Eine Vielzahl von Faktoren beeinflusst die Erfolgschancen für einen vaginalen Entbindungsversuch.

Positive Prädiktoren für den Erfolg eines spontanen Entbindungsversuchs:

- mindestens eine vaginale Entbindung in der Anamnese

Kontraindikationen für eine

vaginale Entbindung [10]

\section{relative Kontraindikationen}

- großes Kind/V.a. Makrosomie (fetomaternale Proportion!)

- 2 oder mehr Sectiones in der Vergangenheit

- Gemini

- Beckenendlage

- unbekannte uterine Schnittführung bei der vorangegangenen Sectio

- maternales Alter $>40$ Jahre

absolute Kontraindikationen

- Sectio-Wunsch der Mutter

- aktuelle Sectio-Indikationen (fetale Indikation, Placenta praevia/Placenta accreta/increta/percreta, maternale Indikation)

- im Vergleich zur vorangegangenen Sectio unveränderte Sectio-Indikation (z. B. mütterliche Erkrankung, Becken-/Uterusanomalie, Myomenukleation eines großen Myoms oder mit Eröffnung des Cavum uteri)

- uteriner T-Schnitt (Uterusrupturrisiko 4-9\%) oder uteriner Längsschnitt (Uterusrupturrisiko 1-7\%)

- Z.n. oder aktuell V.a. Narbendehiszenz oder Uterusruptur (Uterusrupturrisiko 6-32\%)
- primäre Sectio (z.B. wegen Beckenendlage) in der Anamnese bzw. Sectio nicht wegen Geburtsstillstand/Missverhältnis

- Abstand der letzten Sectio zur aktuellen Schwangerschaft > 12 Monate

- (stoffwechsel-) gesunde Patientin, BMI <30

- keine Beckenanomalien/Uterusfehlbildungen

- spontaner Wehenbeginn

- fetales Schätzgewicht <4000 g

\section{Tipp für die Praxis}

Die Geburtsvorbereitung sollte auch die Aufklärung über die erhöhten Risiken einer sekundären Sectio und eines erhöhten Blutverlusts unter der Geburt enthalten.

\section{Primäre (elektive) Re-Sectio}

Die primäre Re-Sectio gilt als gleichwertige Alternative zum vaginalen Entbindungsversuch bei Z.n. Sectio. Bei der Entscheidung über den angestrebten Entbindungsmodus ist zu bedenken, dass eine erneute Sectio auch erneut Risiken für primäre Komplikationen (Blutungen, Organverletzung, Verletzungen des Kindes, Wundheilungsstörungen, Infektionen, Thrombosen/Embolien) mit sich bringt. Zudem ist auch das Risiko für Sekundärkomplikationen in weiteren Schwangerschaften erhöht. Dennoch ist die Sectio caesarea eine für Mutter und Kind sichere Entbindungsalternative.

Indikationen für eine elektive Re-Sectio:

- Wunsch der Mutter

- Vorliegen von Kontraindikationen für eine vaginale Entbindung (s.o.) 


\section{Sekundäre Re-Sectio/Notfall-Sectio}

Wie bei der primären Sectio sollte auch die Indikationsstellung einer sekundären Sectio prinzipiell großzügig gehandhabt werden. Sie ist insbesondere bei ausbleibendem Geburtsfortschritt in der Eröffnungs- oder Austreibungsphase sowie bei Beschwerden im Narbenbereich und immer bei Hinweisen auf eine Ruptur angezeigt. Auch unter der Geburt ist der Wunsch der Mutter den Gegebenheiten entsprechend einzubeziehen.

Indikationen für eine sekundäre Re-Sectio:

- subjektive Beschwerden der Patientin im Narbenbereich/V.a. Uterusruptur

- pathologisches CTG

- protrahierter Geburtsverlauf

- Wunsch der Mutter

\section{Geburtsleitung}

Die Betreuung von vaginalen Entbindungen sollte bei Z.n. Sectio unbedingt an einer entsprechend ausgestatteten Klinik erfolgen, da ausreichende Erfahrungen im
Hebammen- und Ärzteteam sowie eine entsprechende Infrastruktur (Neonatologie, Blutbank, schnelle Herstellung einer Sectio-Bereitschaft) insbesondere bei Auftreten von Komplikationen unbedingt erforderlich und für das Outcome von Mutter und Kind entscheidend sind.

\section{Geburtseinleitung}

Das Risiko für eine Uterusruptur ist bei Verwendung geburtseinleitender Maßnahmen erhöht. Da evidenzbasierte Empfehlungen gegenwärtig nicht existieren und die Studienlage inhomogen ist, stützt sich die Vorgehensweise auf die klinischen Erfahrungen von Experten. In jedem Fall ist die Einleitungsindikation strengstens zu überprüfen.

Sofern möglich, sollte der spontane Wehenbeginn immer abgewartet werden.

Ist eine Einleitung indiziert, muss die Patientin über die Risiken und Chancen ausführlich aufgeklärt werden. Eine Übersicht über die Möglichkeiten geburtseinleitender Maßnahmen bzw. Medikamente gibt Tabelle 3.

\section{Tabelle 3}

Möglichkeiten und Risiken der Geburtseinleitung bei Z.n. Sectio [10].

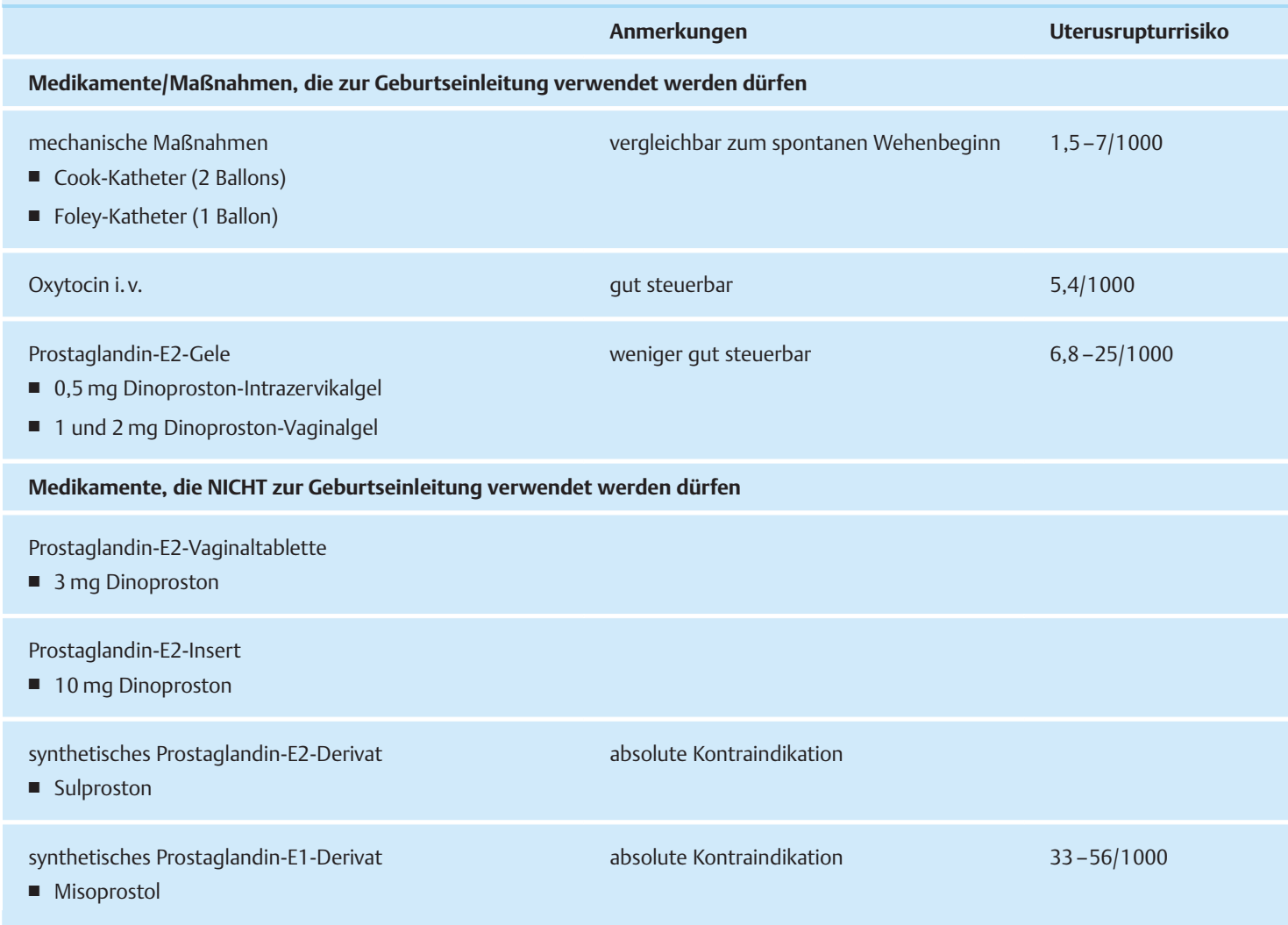


Cave: Synthetische Prostaglandin-Derivate (Sulproston, Misoprostol) sind für die Geburtseinleitung bei Z. n. Sectio absolut kontraindiziert. Wegen des hohen Rupturrisikos (4,5\%) dürfen Oxytocin und Prostaglandine nicht gleichzeitig appliziert werden.

\section{Tipp für die Praxis}

Um einer uterinen Überstimulation schnell entgegenwirken zu können, muss eine Notfalltokolyse ( $\beta 2$ Agonisten, z. B. Fenoterolhydrobromid) bereitliegen. Unter der Einleitung ist eine intensive fetomaternale Überwachung, vor allem mit engmaschiger CTGAbleitung, regelmäßigen Kreislaufkontrollen, Einschätzung der Schmerzen, Wehenfrequenz, Geburtsfortschritt obligat.

\section{Analgesie}

Sofern die CTG-Ableitung sicher ist, können unter der Geburt alle üblichen Medikamente für die Analgesie oder Anästhesie verwendet werden.

Der Z.n. Sectio ist keine Kontraindikation für eine peridurale Anästhesie (PDA).

\section{Danksagung}

Die MRT-Bilder wurden mit freundlicher Genehmigung von der Klinik und Poliklinik für Diagnostische und Interventionelle Radiologie der Universität Leipzig zur Verfügung gestellt.

\section{Kasuistik zum fallorientierten Lernen}

Wir betreuten eine 26-jährige Patientin (5 Gravida, 3 Para) mit Z. n. $1 \times$ Sectio, $2 \times$ Abort und $1 \times$ Spontanpartus. In der aktuellen Schwangerschaft lag, wie in der vorangegangenen Schwangerschaft auch, ein insulinpflichtiger Gestationsdiabetes vor. Anamnestisch waren zusätzlich eine Hypothyreose sowie ein Nikotinabusus nennenswert.

Die Sectio beim 1. Kind war vor 7 Jahren aufgrund eines pathologischen CTGs in der Eröffnungsperiode indiziert. Mittels der Misgav-Ladach-Technik wurde ein $3910 \mathrm{~g}$ schwerer, gesunder Junge entwickelt. Zwei folgende Aborte 1 und 3 Jahre später erforderten keine Kürettage. Für die Geburt des 2. Kindes vor 3 Jahren entschied sich die Patientin für einen spontanen Entbindungsversuch. Dieser war nach einer Geburtseinleitung wegen des insulinpflichtigen Gestationsdiabetes in der 39. SSW erfolgreich und verlief unkompliziert.

Die Erstvorstellung in der aktuellen Schwangerschaft erfolgte in der 32. SSW wegen eines präpathologischen CTGs beim niedergelassenen Gynäkologen. Bei normalen Kontroll-CTG erfolgte eine im weiteren Verlauf unauffällige 14-tägige Betreuung bei Gestationsdiabetes. Die Hinterwandplazenta zeigte sich tiefreichend, bis $12 \mathrm{~cm}$ vor dem inneren Muttermund abgrenzbar. Das untere Uterinsegment war sonografisch intakt und unauf- fällig, mit einer Gesamtdicke von 5,8 mm und einer Myometriumdicke von 2,8 mm in der 36. SSW (Abb. 5). Auf Wunsch der Patientin wurde bei gut eingestelltem Blutzucker und normwertigem sonografischen Gewichtsverlauf wieder ein spontaner Entbindungsversuch geplant. Wegen des Gestationsdiabetes war auch dieses Mal die Geburtseinleitung in der 39. SSW notwendig.

Die Einleitung wurde bei Wohlbefinden der Mutter und bei unauffälligem AufnahmeCTG (Abb. 6) mit 1 mg Dinoproston-Vaginalgel begonnen. Nach Fortsetzung des 1 . Zyklus mit $0,5 \mathrm{mg}$ Dinoproston-Intrazervikalgel und Beginn eines 2. Zyklus mit $2 \mathrm{mg}$ Dinoproston-Vaginalgel kam es schließlich zu
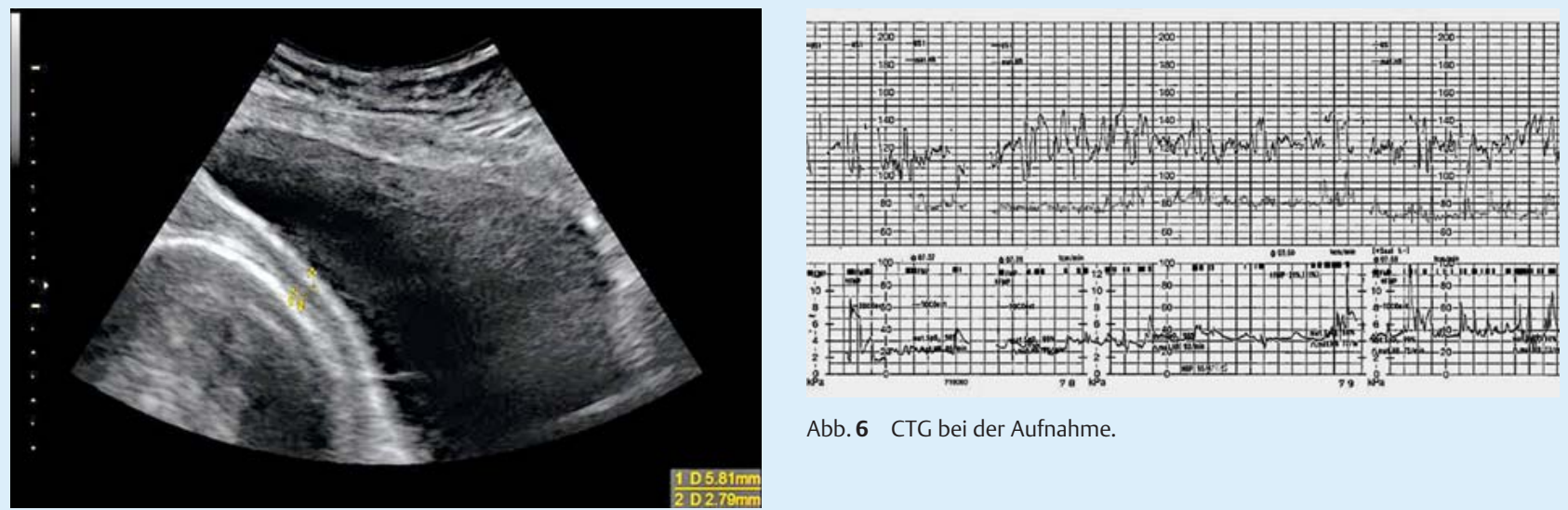

Abb. 6 CTG bei der Aufnahme.

Abb. 5 Transabdominale Sonografie mit Darstellung der Gesamtdicke des unteren Uterinsegments (1) und der Dicke des Myometriums (2). 
Kasuistik zum fallorientierten Lernen (Fortsetzung)

regelmäßigen, kräftigen Wehen. Hierunter eröffnete sich der Muttermund innerhalb von 2 Stunden von 1 auf $3 \mathrm{~cm}$. Für die Schmerztherapie wurden auf Wunsch der Patientin Butylscopolaminiumbromid, Paracetamol und Pethidin verwendet.

Bei einer Muttermundweite von $3 \mathrm{~cm}$ gab die Patientin starke Schmerzen über der Symphyse und Angstgefühl an. Im bis dahin unauffälligen CTG zeigten sich späte Dezelerationen bis 80 spm und ein Abfall der basalen Herzfrequenz von 125 auf 100 spm (Abb. 7). Die kindliche Leitstelle, welche bei der Muttermundweite von $2 \mathrm{~cm}$ noch auf Höhe der oberen Schoßfugenrandebene tastbar war, zeigte sich nun weit oberhalb der Symphyse. Unter dem Verdacht einer Uterusruptur wurde umgehend eine Not-Sectio indiziert. Intraoperativ bestätigte sich das Vorliegen einer Uterusruptur im alten Narbenbereich mit fast vollständiger Lokalisation des kindlichen
Kopfes vor dem Uterus. Es gelang die problemlose Entwicklung eines reifen, lebensfrischen Mädchens mit einem Geburtsgewicht von $2817 \mathrm{~g}$, Apgar (1/5/10 min) 8/9/9, arteriellem Nabelschnur-pH 7,29 und Base Excess (Basenabweichung) - 4,2. Die Plazenta wurde vollständig entwickelt. Die Versorgung der quer verlaufenden, verstärkt blutenden Uterusruptur erfolgte unkompliziert mittels Einzelknopfnähten. Postpartum traten keine Besonderheiten auf. Bei einem Kontroll-Hb von $6,30 \mathrm{mmol} / \mathrm{l}$ war keine Bluttransfusion notwendig. Nach regelrechtem postoperativem Verlauf wurde die Patientin gemeinsam mit ihrem Kind am 4. Tag nach der Sectio aus der stationären Betreuung entlassen.

\section{Beurteilung}

Der Fall demonstriert, dass eine Uterusruptur ein unvorhergesehenes, plötzliches Ereignis ist. Nicht selten sind alle pränatal erhobenen Befunde, einschließlich der Ausmessung des unteren Uterinsegmentes, unauffällig. Die
Leitsymptome pathologisches CTG und Schmerzen der Frau sind bei Z. n. Sectio hinweisend auf eine mögliche Uterusruptur. Sie erfordern umgehend die Re-Sectio. Da die Notwendigkeit der Geburtseinleitung ein entscheidender Risikofaktor ist, sollte nach Möglichkeit auf geburtseinleitende Methoden verzichtet werden.

Wie aber die Mehrzahl der betreuten Patientinnen zeigt, ist das Auftreten einer Uterusruptur, auch nach Geburtseinleitung, eine Rarität. Bei der Betreuung von Patientinnen mit Z.n. Sectio muss die Möglichkeit einer sofortigen Sectio-Bereitschaft sowie einer optimalen, sofortigen neonatalen Betreuung gewährleistet sein. Dies und die ausreichende Erfahrung im Ärzte- und Hebammenteam sind entscheidend für das Outcome von Mutter und Kind. Deshalb sollte die Betreuung von Patientinnen mit Z.n. Sectio an einem Perinatalzentrum erfolgen.
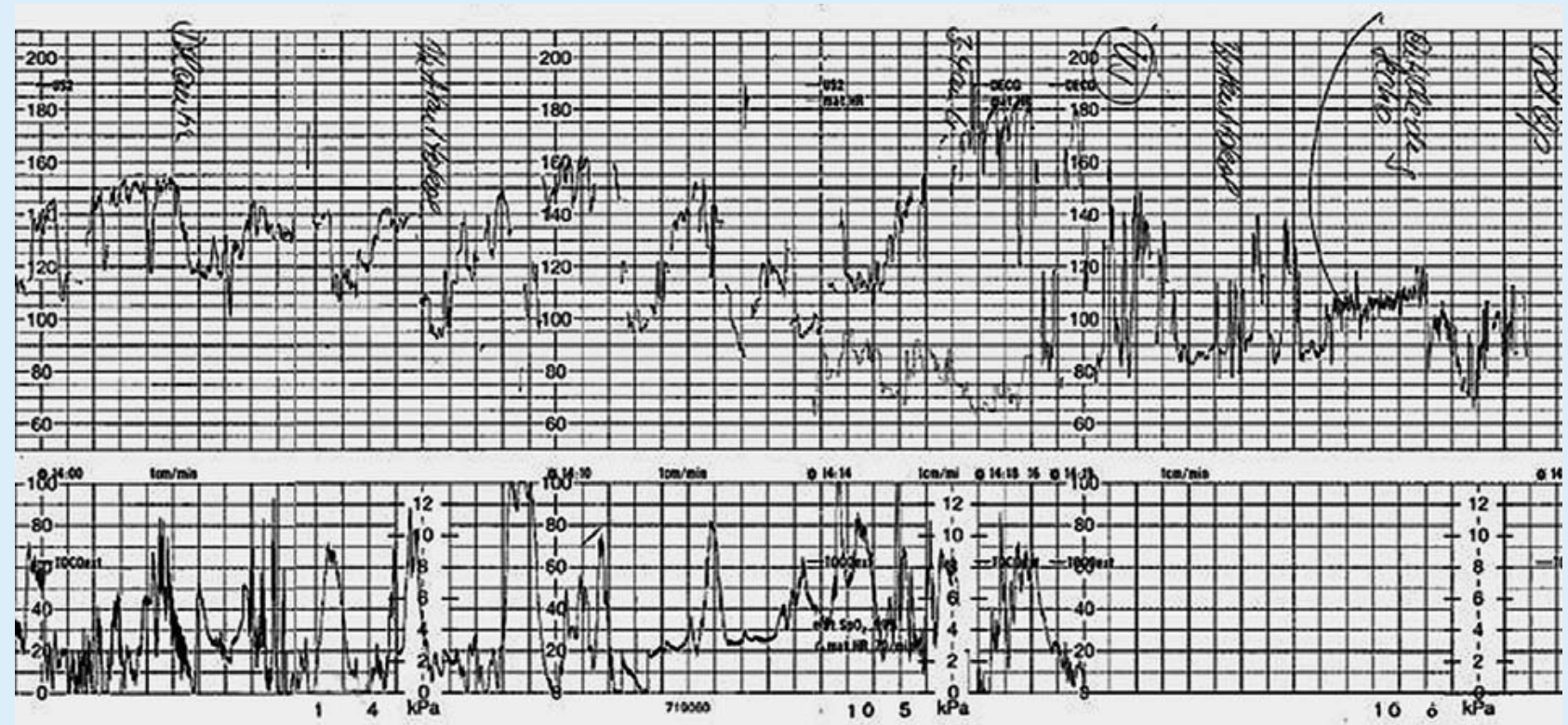

Abb. 7 CTG mit späten Dezelerationen sowie Abfall der basalen Herzfrequenz. 


\section{Kernaussagen}

Der Z.n. Sectio stellt höchste Anforderungen an die behandelnden Gynäkologen/Geburtsmediziner in der ambulanten und auch in der stationären Versorgung. Sekundärkomplikationen durch vorangegangene Sectiones sind zwar selten, aber lebensbedrohlich für Mutter und Kind. Für ein bestmögliches Outcome von Mutter und Kind ist die aufmerksame pränatale Diagnostik deshalb bereits ab der Frühschwangerschaft unverzichtbar. Hierbei ist ein besonderer Blick auf die Implantationsstelle, die Plazentalokalisation und gegebenenfalls auf die Invasionstiefe der Plazenta zu richten. Bei unklaren oder auffälligen Befunden ist eine Überweisung an ein Perinatalzentrum zwingend notwendig. Bei unauffälligen Verläufen sollte die Patientin für die Geburtsplanung in der 35. -36. SSW an ein entsprechendes Perinatalzentrum überwiesen werden. Durch eine geeignete Infrastruktur sind hier alle Möglichkeiten gegeben, auftretende Komplikationen zu beherrschen.

Im gesamten Schwangerschaftsverlauf ist die Aufklärung der Patientin ein wichtiger Bestandteil der Betreuung. Individuell angepasst, muss die Patientin über mögliche Komplikationen und die entsprechenden Symptome informiert sein. Sie muss auch in die Entscheidung über den geplanten Geburtsmodus einbezogen werden. Hierfür ist eine ausführliche Beratung zu den Möglichkeiten und den jeweiligen Risiken durchzuführen. Bei Fehlen von Kontraindikationen ist letztlich die Patientin Entscheidungsträgerin des geplanten Entbindungsmodus.

\section{Über die Autoren}

\section{Janine Hoffmann}

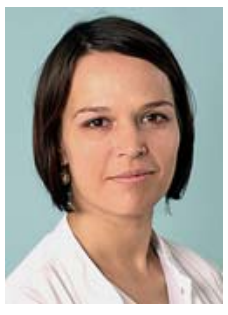

Dr. med. Jahrgang 1979. Studium der Humanmedizin an der Humboldt-Universität zu Berlin. 2011 Promotion. Bis 2011 Weiterbildung in der Abteilung für diagnostische und interventionelle Radiologie Universität Leipzig - Herzzentrum. 2011-2012 Weiterbildung in der Klinik für Gynäkologie und Geburtshilfe, Asklepios Klinik Weißenfels. Seit 2012 Weiterbildung in der Universitätsfrauenklinik Leipzig, bis 2015 Ärztin in der Abteilung für Geburtsmedizin, seit 2015 Ärztin in der Klinik und Poliklinik für Frauenheilkunde. Wissenschaftlicher Schwerpunkt: Bildgebung in der Geburtsmedizin, insbesondere bei Z.n. Sectio und Beckenendlage.

\section{Holger Stepan}

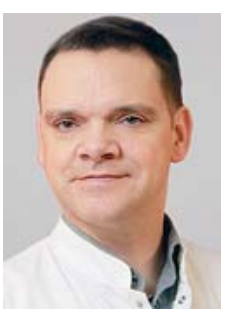

Prof. Dr. med. Jahrgang 1967. Studium der Humanmedizin an der Universität Leipzig. Promotion 1995 über Dopplersonografie bei drohender Frühgeburt. 2000 Facharztanerkennung Gynäkologie und Geburtshilfe; 2001 Habilitation. Seit 2007 Leiter der selbstständigen Abteilung für Geburtsmedizin des Universitätsklinikums Leipzig. Klinische und wissenschaftliche Schwerpunkte: Pränataldiagnostik, Dopplersonografie, hypertensive Schwangerschaftskomplikationen, Plazentainsuffizienz.

\section{Interessenkonflikt}

Die Autoren geben an, dass bezüglich der Veröffentlichung des Manuskripts kein Interessenkonflikt besteht.

\section{Korrespondenzadresse}

Dr. med. Janine Hoffmann

Universitätsfrauenklinik Leipzig

Abteilung für Geburtsmedizin

Liebigstraße 20a

04103 Leipzig

E-Mail: janine.hoffmann@medizin.uni-leipzig.de 


\section{Literatur}

1 Abalos E, Addo V, Brocklehurst P et al. Caesarean section surgical techniques (CORONIS): a fractional, factorial, unmasked, randomised controlled trial. Lancet 2013; 382: 234-248

2 Hemminki E, Meriläinen J. Long-term effects of cesarean sections: ectopic pregnancies and placental problems. Am J Obstet Gynecol 1996; 174: $1569-1574$

3 Jurkovic D, Hillaby K, Woelfer B et al. Cesarean scar pregnancy. Ultrasound Obstet Gynecol 2003; 21: 310

4 Seow KM, Huang LW, Lin YH et al. Cesarean scar pregnancy: issues in management. Ultrasound Obstet Gynecol 2004; 23: $247-253$

5 Ash A, Smith A, Maxwell D. Caesarean scar pregnancy. BJOG 2007; 114: $253-263$

6 Wu X, Xue X, Wu X et al. Combined laparoscopy and hysteroscopy vs. uterine curettage in the uterine artery embolization-based management of cesarean scar pregnancy: a cohort study. Int J Clin Experimental Med 2014; 7: 2793-2803

7 Xiao J, Zhang S, Wang F et al. Cesarean scar pregnancy: noninvasive and effective treatment with high-intensity focused ultrasound. Am J Obstet Gynecol 2014; 211: 356.e1-356.e7

8 Ananth CV, Smulian JC, Vintzileos AM. The association of placenta previa with history of cesarean delivery and abortion: a metaanalysis. Am J Obstet Gynecol 1997; 177: 1071 -1078

9 Belfort MA. Placenta accreta. Am J Obstet Gynecol 2010; 203 : 430-439

10 Deutsche Gesellschaft für Gynäkologie und Geburtshilfe e. V. Leitlinien, Empfehlungen, Stellungnahmen (Stand 2010): Schwangerenbetreuung und Geburtseinleitung bei Zustand nach Kaiserschnitt. AWMF 015/021 (S1)

11 Mar WA, Berggruen S, Atueyi $U$ et al. Ultrasound imaging of placenta accreta with MR correlation. Ultrasound Q 2015; 31: 23 33

12 Deutsche Gesellschaft für Gynäkologie und Geburtshilfe e. V. Leitlinien, Empfehlungen, Stellungnahmen (Stand 2010): Plazentationsstörungen bei Status nach Sectio. Risk-Management zur Vermeidung von Müttersterbefällen. AWMF 015/046

13 Riteau A, Tassin M, Chambon G et al. Accuracy of ultrasonography and magnetic resonance imaging in the diagnosis of placenta accreta. PloS One 2014; 9: e94866
14 D'Antonio F, lacovella C, Bhide A. Prenatal identification of invasive placentation using ultrasound: systematic review and metaanalysis. Ultrasound Obstet Gynecol 2013; 42: 509-517

15 D’Antonio F, lacovella C, Palacios-Jaraquemada J et al. Prenatal identification of invasive placentation using magnetic resonance imaging: systematic review and meta-analysis. Ultrasound Obstet Gynecol 2014; 44: 8-16

16 Meng X, Xie L, Song W. Comparing the diagnostic value of ultrasound and magnetic resonance imaging for placenta accreta: a systematic review and meta-analysis. Ultrasound Med Biol 2013; 39: $1958-1965$

17 Valentin L. Prediction of scar integrity and vaginal birth after caesarean delivery. Best Pract Res Clin Obstet Gynaecol 2013; 27 : 285-295

18 Rozenberg P, Goffinet F, Philippe $\mathrm{HJ}$ et al. Thickness of the lower uterine segment: its influence in the management of patients with previous cesarean sections. Eur J Obstet Gynecol Reprod Biol 1999; 87: 39-45

19 Kok N, Wiersma IC, Opmeer BC et al. Sonographic measurement of lower uterine segment thickness to predict uterine rupture during a trial of labor in women with previous Cesarean section: a meta-analysis. Ultrasound Obstet Gynecol 2013; 42: 132-139

20 Jastrow N, Antonelli E, Robyr R et al. Inter- and intraobserver variability in sonographic measurement of the lower uterine segment after a previous Cesarean section. Ultrasound Obstet Gynecol 2006; 27: 420-424

21 Rozenberg P, Goffinet F, Phillippe HJ et al. Ultrasonographic measurement of lower uterine segment to assess risk of defects of scarred uterus. Lancet 1996; 347: $281-284$

22 Landon MB, Hauth JC, Leveno KJ et al. Maternal and perinatal outcomes associated with a trial of labor after prior cesarean delivery. N Engl J Med 2004; 351: 2581 -2589

23 Lydon-Rochelle M, Holt VL, Easterling TR et al. Risk of uterine rupture during labor among women with a prior cesarean delivery. N Engl J Med 2001; 345: 3-8

24 Tahseen S, Griffiths M. Vaginal birth after two caesarean sections (VBAC-2)-a systematic review with meta-analysis of success rate and adverse outcomes of VBAC-2 versus VBAC-1 and repeat (third) caesarean sections. BJOG 2010; 117: 5-19 


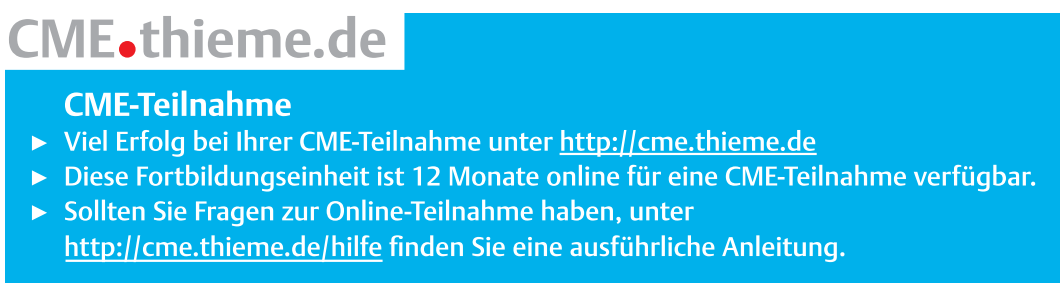

\section{CME-Fragen}

1 Für die Geburtsplanung bei Z.n. Sectio ist folgende Aussage richtig?

A Eine Geburtsplanung ist immer notwendig.

B Es muss eine primäre Re-Sectio geplant werden.

C Die sonografische Messung des unteren Uterinsegments ist notwendig.

D Die sonografische Messung des unteren Uterinsegments muss transvaginal erfolgen.

E Eine MRT-Untersuchung ist notwendig.

Welche Maßnahme ist nach Mutterschaftsrichtlinien für die Planung einer spontanen Geburt bei Z. n. Sectio nicht gefordert?

A eine Anamnese über den Verlauf aller vorangegangenen Schwangerschaften

B die klinische Untersuchung der Patientin

C die sonografische Schätzung des Kindsgewichtes

D die Ausmessung des Beckenringes mit dem Beckenzirkel

E die sonografische Bestimmung der Plazentalokalisation

3 Welche Aussage zu Plazentationsstörungen ist richtig?

A Bei V.a. Vorliegen einer Plazentationsstörung muss eine möglichst frühe Anbindung an ein Perinatalzentrum erfolgen.

B Die Placenta accreta ist keine echte Plazentationsstörung.

C Bei der Placenta increta können benachbarte Organe infiltriert sein.

D Blutungen sind bei vorliegender Placenta percreta selten.

E Bei einer Placenta accreta ist das Blutungsrisiko gering.

\section{Welche Antwort ist falsch?}
A Uterusrupturen sind selten.
B Eine Uterusruptur tritt am häufigsten bei Patientinnen mit Z. n. Sectio auf.
C Eine Uterusdehiszenz ist für den Feten und die Mutter immer lebensgefährlich.
D Uterusdehiszenzen werden häufig erst zufällig intraoperativ entdeckt.
E Bei V.a. Uterusdehiszenz ist eine Re-Sectio indiziert.

\section{Welche Aussage trifft zur Sonografie des unteren Uterinsegments bei Z.n. Sectio zu?}

A Sie ist eine gut validierte Methode zur Beurteilung der Uterotomienarbe und obligater Bestandteil der Geburtsplanung bei Z.n. Sectio.

B Mit dieser Methode ist es möglich, die Festigkeit der Uterotomienarbe einzuschätzen.

C Das Verfahren kann transabdominal und transvaginal erfolgen.

D Mit dieser Methode lässt sich der Erfolg eines geplanten Spontanpartus sicher voraussagen.

E Bei einer Dicke des gesamten unteren Uterinsegments $<4 \mathrm{~mm}$ darf keine spontane Entbindung geplant werden.

A Nach initial starken Wehen hören die Wehen schlagartig auf.

B Die Patientin verspürt stärkste Schmerzen.

C Die sogenannte Bandl'sche Furche wird erkennbar.

D Häufig treten vaginale Blutungen auf.

E Symptome sind meist gering oder fehlen völlig. 
7 Welche Aussage trifft zu?

A Oxytocin darf bei Z.n. Sectio unter der Geburt nicht verwendet werden.

B Das synthetische Prostaglandin-E1-Derivat Misoprostol darf bei Z. n. Sectio uneingeschränkt für die Geburtseinleitung eingesetzt werden.

C Das synthetische Prostaglandin-E2-Derivat Sulproston darf bei Z. n. Sectio uneingeschränkt für die Geburtseinleitung eingesetzt werden.

D Eine PDA ist bei Z.n. Sectio unter der Geburt kontraindiziert.

E Die geburtshilfliche Schmerzmedikation ist bei Z.n. Sectio unter der Geburt uneingeschränkt möglich.

8 Welche Aussage ist falsch? Nach gültigen Mutterschaftsrichtlinien gelten für die Planung einer spontanen Entbindung folgende relative Kontraindikationen:
A Z.n. Schulterdystokie
B Gemini
C Beckenendlage
D Zervixinsuffizienz
E Z.n. $2 \times$ Sectio in der Vergangenheit

9 Welche Aussage ist falsch? Nach gültigen Mutterschaftsrichtlinien gelten für die Planung einer spontanen Entbindung folgende absolute Kontraindikationen:
A Sectio-Wunsch der Mutter
B vorzeitiger Blasensprung (<37. SSW)
C Placenta praevia
D Z.n. uterinem T-Schnitt
E V.a. Narbendehiszenz/Uterusruptur

Eine Patientin (Gravida 2, Para 1) mit Z.n. Sectio stellt sich zur Geburtsplanung vor. Welcher der erhobenen Risikofaktoren ist kein positiver Prädiktor für den Erfolg eines spontanen Entbindungsversuches?
A adipöse Patientin mit insulinpflichtigem Diabetes mellitus
B $1 \times$ Spontanpartus in der Anamnese
C Z.n. $1 \times$ Sectio, nicht wegen eines Geburtsstillstandes oder eines Missverhältnisses
D keine bekannten Beckenanomalien
E fetales Schätzgewicht $<4000 \mathrm{~g}$, Schädellage 